\title{
GENOTIPOS DE FRIJOL (Phaseolus vulgaris L.) RESISTENTES A Xanthomonas campestris pv. phaseoli DE MEXICO1
}

\author{
Rosa Navarrete ${ }^{2}$, Jorge A. Acosta ${ }^{3}$
}

\begin{abstract}
RESUMEN
Genotipos de frijol (Phaseolus vulgaris L.) resistentes a Xanthomonas campestris pv. phaseoli de México. Durante 1995 se evaluó la reacción de genotipos de frijol de diversos origenes a $X c p$, bajo condiciones de invernadero en el Campo Experimental del Valle de México, del INIFAP. Se realizaron tres experimentos con a) 120 , b) 44 y c)siete genotipos de frijol. Las plantas se inocularon por corte con navajas en la etapa $V_{3}$, a y b con una mezcla de nueve cepas de $X c p$ y el c, con cada una de siete cepas con diferente grado de patogenicidad. La severidad se evaluó 20 días después de la inoculación, por comparación con una escala visual de nueve grados. Los datos se analizaron bajo un diseño completamente al azar. En a, los genotipos que mostraron reacción de resistencia a Xcp fueron: A 36, A 475, G 5686, G 11867, Harowood, SEA 14, XAN 266, MCD 4012 y REN 27. En b los genotipos resistentes fueron: Sequía Durango, Taylor y XAN 30. En los experimentos anteriores la severidad de la enfermedad mostró una distribución normal, con el máximo número de genotipos en el grado de severidad cinco en a y seis en b. Los resultados obtenidos indican que el uso de mezclas de cepas de bacterias con diferente patogenicidad es eficiente para identificar genotipos de frijol resistentes a $X c p$. Los genotipos resistentes identificados en el último experimento, mostraron respuesta diferencial e interacciones genotipo por cepa. REN 27 y SEA 14 mostraron resistencia a las cepas utilizadas.
\end{abstract}

\begin{abstract}
Common bean genotypes (Phaseolus vulgaris L.) resistant to isolates of Xanthomonas campestris pv phaseoli from Mexico. During 1995 the reaction of bean genotypes from different origin to $X c p$ was scored under greenhouse conditions at the Valle de Mexico Experimental Station of INIFAP. Three experiments were made with a)120, b) 44 and c) seven bean genotypes. Plants of a and b at the $V_{3}$ stage were inoculated with razor blades using a mixture of nine $X c p$ strains, and c individually with seven different strains of $X c p$ with different grade of virulence. The disease severity was scored 20 days after the inoculation comparing with a visual scale of nine grades. Data were analyzed under a completely random design. In a the genotypes that showed resistant reaction were: A 36, A 475, G 5686, G 11867, Harowood, SEA 14, XAN 266, MCD 4012 and REN 27. In $\mathrm{b}$ the resistant genotypes were: Sequía Durango, Taylor and XAN 30. The disease severity of all genotypes in each experiment showed a normal distribution, with the maximum number of genotypes at the five grade of severity in the first trial and six in the second. The resistant genotypes in the last trial showed a differential reaction and genotype by strain interaction, REN 27 and SEA 14 proved to be resistant to most of the strains inoculated. These results indicate that the inoculation of mixtures of $X c p$ strains with different pathogenicity was efficient to identify resistant bean genotypes.
\end{abstract}

\section{INTRODUCCIÓN}

El tizón común inducido por Xanthomonas campestris pv. phaseoli (Smith) Dye (Xcp) es una enfermedad importante del frijol común (Phaseolus vulgaris L.), debido a los daños que ocasiona y a su amplia distribución en México, principalmente en las áreas productoras del Altiplano, en donde se ubica dentro de los primeros cuatro problemas fitopatológicos (López,
1991). Las pérdidas en el rendimiento ocasionadas por esta enfermedad pueden variar entre el 15 y el $30 \%$ (Valle et al., 1987; Campos, 1987), dependiendo de las condiciones climáticas durante el ciclo biológico del frijol, de la variedad que se cultive y de la concentración del inóculo existente.

$X c p$ es transmitida por semilla (Sheppard et al., 1989) y en México la mayoría de los productores, prin-

\footnotetext{
1 Presentado en la XLV Reunión Anualdel PCCMCA en Guatemala. 1999

2 UNIGRAS, FES-Cuautitlán, UNAM, Apdo. Postal 25. Cuautitlán Izcalli, Edo. de México, C.P. 57400.

3 CEVAMEX-INIFAP, Apdo. Postal 10, Texcoco, Edo. de México, C.P. 56230.
} 
cipalmente de las zonas temporaleras y de escasos recursos, utilizan la semilla que ellos mismos producen para establecer el siguiente ciclo agrícola (Acosta, 1992). Esto contribuye a la dispersión de la bacteria, a la ocurrencia de variantes de la bacteria en una área específica y al desarrollo de la enfermedad.

Entre las medidas recomendadas para evitar el daño causado por Xcp se encuentran la rotación de cultivos, la eliminación de residuos de la cosecha, la modificación de las fechas de siembra, el ajuste de la densidad de siembra, la aplicación de productos a base de cobre, el uso de antibióticos y el uso de genotipos resistentes (Saettler, 1991; Gilbertson y Maxwell, 1992; Singh, 1992).

En diversos países se han identificado genotipos resistentes al tizón común (Coyne y Schuster, 1973; Singh, 1992), pero por lo general, éstos no se han adaptado a las condiciones del Altiplano de México o no han mostrado resistencia a las cepas locales de la bacteria. En México también se han identificado algunos genotipos resistentes al tizón común (Crispín et al., 1976; Lépiz, 1979), pero al igual que en los otros casos no han mostrado estabilidad en la resistencia al sembrarse en regiones diferentes a donde fueron identificados como resistentes.

Los objetivos de este trabajo fueron: I) identificar genotipos de frijol ( $P$. vulgaris) resistentes al tizón común inducido por Xanthomonas campestris pv. phaseo$l i$ en un grupo de genotipos mejorados, de diferentes orígenes, II) verificar la resistencia en otro grupo de genotipos clasificados como resistentes a esta enfermedad y III) determinar la interacción en la reacción a siete cepas de Xanthomonas campestris pv. phaseoli en siete genotipos de frijol.

\section{MATERIALES Y MÉTODOS}

Para cubrir los objetivos planteados en esta investigación se realizaron tres experimentos:

1. Caracterizar la reacción a la inoculación con una mezcla de cepas de Xcp, en un grupo de 120 genotipos mejorados de frijol de diversos orígenes y línas mejoradas del CIAT, que mostraron resistencia a la infección natural por antracnosis y tizón común en el Campo Experimental del Valle de México, en el ciclo primavera-verano 1994.

2. Confirmar la reacción de resistencia a la inoculación con una mezcla de cepas de $X c p$ en un grupo de 44 genotipos de frijol considerados como resistentes al tizón común en otros experimentos; ade- más se incluyeron los genotipos Flor de Mayo Bajío y Porrillo Sintético como testigos susceptibles.

3. Verificar la reacción a la inoculación con cada una, de siete cepas de $X c p$, en un grupo de siete genotipos de frijol considerados como resistentes al tizón común en otros experimentos. Los genotipos fueron previamente evaluados por inoculación con la mezcla de cepas de Xcp.

Las condiciones de coducción de los tres experimentos fueron similares en los aspectos de siembra, cuidado e inoculación de las plantas.

\section{Sitio experimental.}

Los experimentos se realizaron en el invernadero del Campo Experimental del Valle de México (CEVAMEX-INIFAP), Texcoco, Edo. de México (19²9’N, $98^{\circ} 51^{\prime}$ O y $\left.2240 \mathrm{msnm}\right)$.

\section{Siembra de plantas.}

Las plantas se cultivaron en macetas, las que se llenaron con $1.5 \mathrm{~kg}$ de una mezcla de suelo y arena (2:1), esterilizada con bromuro de metilo, se humedecieron y se conservaron en invernadero. De cada variedad utilizada se sembraron cuatro semillas de frijol por maceta. Cuando las plantas llegaron a la etapa fenológica $\mathrm{V}_{3}$ se inocularon. Durante la conducción del experimento se suministró a las plantas el riego necesario y se realizó el control de plagas mediante la aplicación de agroquímicos y con trampas adhesivas amarillas.

\section{Inoculación.}

El inóculo se preparó mezclando nueve cepas de $X c p$ con diferente grado de patogenicidad. Las cepas se obtuvieron de frijol infectado en varias localidades de México (Cuadro 1). Las bacterias se cultivaron en medio YDC, se incubaron a $28^{\circ} \mathrm{C}$ durante 48 horas

Cuadro 1. Características de las cepas de X. campestris pv. phaseoli utilizadas en los experimentos.

\begin{tabular}{llll}
\hline Cepa & \multicolumn{1}{c}{ Origen } & Aislada de & Patovar \\
\hline Mex-1 & Pabellón, Ags. & Semillas & Xcp \\
Mex-24 & Durango, Dgo. & Semillas & Xcp \\
Mex-27 & Durango, Dgo. & Semillas & Xcp \\
Mex-44 & Cd. Cuauhtémoc, Chih. & Semillas & Xcp \\
Mex-13 & Chapingo, Mex. & Semillas & Xcp \\
Mex-17 & Chapingo, Mex. & Hojas & Xcp \\
Mex-60 & Guadalupe Sabinal, Pue. & Hojas & Xcp \\
Mex-99 & Chapingo, Mex. & Hojas & Xcp \\
Mex-95 & Guadalupe Sabinal, Pue. & Hojas & Xcp \\
\hline
\end{tabular}

$\mathrm{Xcp}=X$. campestris pv. phaseoli. 
(Sheppard et al., 1989) y se preparó una suspensión acuosa de las bacterias a una concentración de $3 \times 10^{7}$ ufc, la cual se determinó en base a la escala de McFarland (Kiraly et al., 1970). Las inoculaciones se realizaron por corte con navajas, en el borde de cada foliolo de plantas en la etapa $V_{3}$ se hicieron dos cortes transversales con navajas paralelas, separadas entre sí un centímetro y se humedeció la zona herida con la suspensión de las bacterias (Pastor-Corrales et al., 1981).

\section{Condiciones de incubación.}

Después de la inoculación las plantas se conservaron durante 48 horas a una temperatura de $26-28{ }^{\circ} \mathrm{C}$, con una humedad relativa mayor de $90 \%$, con 12 horas de luz y 12 de obscuridad. Posteriormente se conservaron en condiciones normales de invernadero (temperatura similar y humedad relativa cercana al 70\%), hasta que se evaluó la severidad de la enfermedad.

\section{Evaluación de la severidad.}

La reacción de severidad se evaluó en la hojas inoculadas a los 20 días después de la inoculación, en base a una escala visual de nueve grados, donde uno es tejido sano y nueve tejido muerto (CIAT, 1987). En esta escala las plantas con calificación de uno a tres se consideran resistentes, de cuatro a seis de reacción intermedia y de siete a nueve susceptibles.

\section{Análisis de los datos.}

Los datos obtenidos se analizaron por medio del programa estadístico para microcomputadoras MSTATC (Freed et al., 1991) y se realizó la Prueba Múltiple de Duncan para la separación de medias.

\section{RESULTADOS}

\section{Experimento I.}

El análisis de varianza indicó que las diferencias en la reacción de los genotipos de frijol a la inoculación con la mezcla de nueve cepas de $X c p$ fueron estadísticamente significativas $(\mathrm{P}<0,05)$.

En este experimento destacaron los genotipos A 36, A 475, G 5686, G 11867, Harowood, SEA 14, XAN 266, MCD 4011, MCD 4012 y REN 27, los que mostraron resistencia a una amplia gama de patogenicidad de la bacteria, debido a que fueron inoculados con una mezcla de nueve cepas de $X c p$ (Cuadro 2). Como ya se había mencionado estos genotipos habían mostrado resistencia a la infección natural por antracnosis y tizón común en campo durante el ciclo agrícola de 1994.
En evaluaciones de la reacción de un grupo de genotipos al tizón común conducidas en Colombia, los genotipos A 475, A 483, RAB 583, RAB 50 y AND 1007 resultaron susceptibles (Rodríguez et al., 1995). A diferencia de los resultados obtenidos en esta investigación en donde la línea A 475 fue resistente, A 483, AND 1007 y RAB 583 tuvieron reacción intermedia y RAB 50 resultó susceptible. Mientras que los genotipos COS 16 y RAB 590 tuvieron una reacción intermedia en ambos estudios. Las diferencias entre los resultados de este experimento y los de Rodríguez et al. (1995) pudieron ser ocasionadas porque se utilizaron cepas de Xcp con distinta patogenicidad y porque los experimentos se desarrollaron bajo diferentes condiciones culturales.

La respuesta de los genotipos a la inoculación con una mezcla de cepas de Xcp con diferentes grados de patogenicidad, mostró una distribución normal ( Fig. 1), con el máximo número de genotipos en el grado de severidad cinco, esta figura sugiere la presencia de resistencia de tipo horizontal dentro de este grupo de genotipos. Los genotipos resistentes podrían aprovecharse en futuros programas de resistencia contra el tizón común.

\section{Experimento II.}

A pesar de que los genotipos utilizados en este experimento fueron seleccionados por la resistencia que han mostrado en otras investigaciones (CIAT, 1984; CIAT, 1988), 31 de los 44 genotipos resultaron susceptibles al ser inoculados con la mezcla de cepas de Xcp de México, excepto Sequía Durango, Taylor y XAN 30 que resultaron resistentes. Estos genotipos podrían considerarse con resistencia a una amplia gama de patogenicidad de la bacteria, ya que se inocularon con una mezcla de nueve cepas de Xcp con distinta patogenicidad. El análisis de varianza indicó que las diferencias en la respuesta a la inoculación con Xcp fueron estadísticamente significativas $(\mathrm{P}>0,05)$.

La gráfica formada con las reacciones de resistencia a la mezcla de cepas de Xcp mostró una apariencia de distribución normal, con el máximo número de genotipos en el grado seis de severidad, es decir con reacciones de tipo intermedio (Fig. 2). Aún cuando las frecuencias de los genotipos formaron dos picos, en general las reacciones de severidad del grado cinco al nueve parecen de tipo cuantitativo, lo cual sugiere la presencia de resistencia de tipo horizontal dentro del grupo de genotipos evaluados, esto deberá confirmarse al realizar los estudios genéticos del hospedante.

Los genotipos que no mostraron resistencia a las cepas de México, pero que se han reconocido como resistentes en otros lugares, probablemente tengan un fuerte componente de resistencia vertical, como lo 
Cuadro 2. Características de genotipos del experimento I identificados como resistentes a la inoculación con una mezcla de nueve cepas de Xanthomonas campestris pv. phaseoli en invernadero reacción de severidad al tizón común.

\begin{tabular}{|c|c|c|c|c|c|}
\hline Genotipo & Origen & $\begin{array}{c}\text { Color de } \\
\text { grano }\end{array}$ & $\begin{array}{l}\text { Hábito de } \\
\text { crecimiento }\end{array}$ & $\begin{array}{c}\text { Estatus } \\
\text { Biológico }\end{array}$ & Severidad \\
\hline San Cristóbal 83 & Venezuela & Rojo & & Criollo & $3,7 *$ \\
\hline G 76 & USA (Red Kloud) & Rosado & I & Criollo & 3,7 \\
\hline 83B 342 & & & & & 3,7 \\
\hline APN 114 & CIAT, Colombia & & & Mejorado & 3,7 \\
\hline SEA 13 & CIAT, Colombia & Blanco & & Mejorado & 3,5 \\
\hline A 193 & CIAT,Colombia & Rojo moteado & II & Mejorado & 3,5 \\
\hline TF 9453 & & & & & 3,5 \\
\hline MAM 28 & CIAT, Colombia & Crema-beige & III & Mejorado & 3,5 \\
\hline MCD 4014 & CIAT, Colombia & Crema-beige moteado & III & Mejorado & 3,5 \\
\hline Bola 60 días & Perú & Crema-beige & I & Criollo & 3,5 \\
\hline СР 006 92-4 & & & & & 3,2 \\
\hline Taylor & USA & Rojo & I & Mejorado & 3,2 \\
\hline ICA CERINZA & CIAT, Colombia & Blanco & & Mejorado & 3,2 \\
\hline XAN 297 & CIAT, Colombia & Rojo & IIA & Mejorado & 3,2 \\
\hline DICTA 17 & Honduras & & & Mejorado & 3,2 \\
\hline XR 17419 & & & & & 3,2 \\
\hline XAN 266 & CIAT, Colombia & Negro & & Mejorado & 3,0 \\
\hline A 475 & CIAT, Colombia & Morado moteado & I & Mejorado & 3,0 \\
\hline A 36 & CIAT, Colombia & & & Mejorado & 3,0 \\
\hline G 11867 & Holanda & Crema-beige & I & Criollo & 3,0 \\
\hline G 5686 & Ecuador & Café-marrón & I & Criollo & 2,7 \\
\hline REN 27 & CIAT, Colombia & Blanco & IIA & & 2,7 \\
\hline SEA 14 & CIAT, Colombia & Blanco & & Mejorado & 2,5 \\
\hline Harowood & Canadá & Blanco & & Mejorado & 2,5 \\
\hline MCD 4011 & CIAT, Colombia & Crema-beige moteado & I & Mejorado & 2,5 \\
\hline MCD 4012 & CIAT, Colombia & Crema-beige moteado & III & Mejorado & 2,5 \\
\hline Flor de Mayo Bajío ${ }^{1}$ & & & & Mejorado & 8,3 \\
\hline Promedio $(n=120)$ & & & & & 4,8 \\
\hline Amplitud de reacción & & & & & $2,5-8,3$ \\
\hline
\end{tabular}

DMS $(0,05)=1.360, *$ media de cuatro repeticiones, 1-3 resistente, 4-6 intermedio y 7-9 susceptible, SEA = variedad avanzada de sequía, $\mathrm{XAN}=$ proyecto Xanthomonas, $\mathrm{MCD}=$ gen I dominante de virus mosaico común del frijol, REN = proyecto potencial de rendimiento. 1 testigo susceptible.

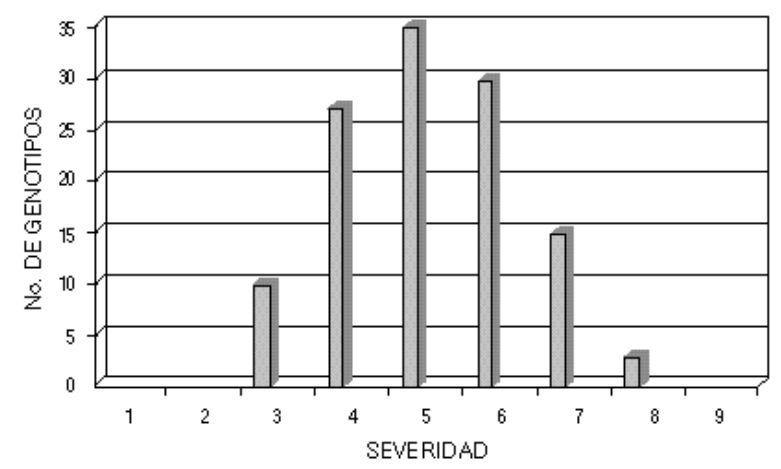

Figura 1. Reacción de genotipos de P. vulgaris a la inoculación con una mezcla de nueve cepas de Xanthomonas campestris pv. phaseoli. México, 1995.

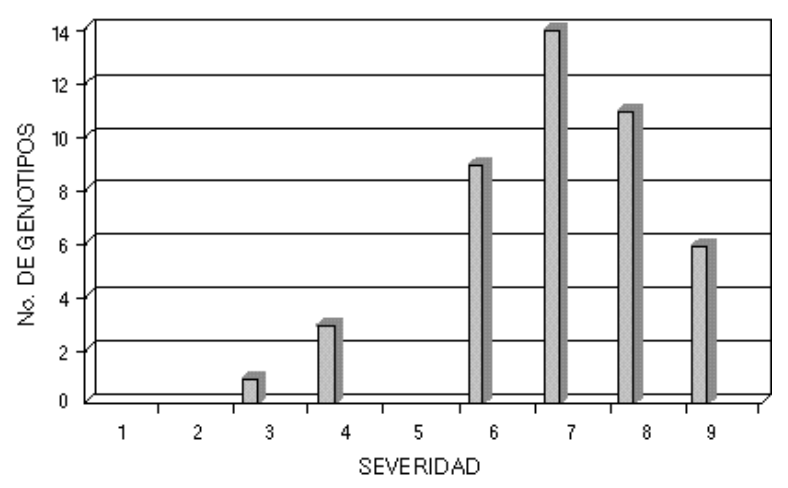

Figura 2. Reacción de genotipos de $P$. vulgaris (resistentes) a la inoculación con una mezcla de nueve cepas de Xanthomonas campestris pv. phaseoli. México, 1995. 
sugiere el hecho de que en esta investigación hayan resultado susceptibles. Además, muchos de esos genotipos han obtenido su resistencia de una o dos fuentes genéticas, como la variedad PI 207262 (Schuster et al., 1973) y de genotipos de P. acutifolius (Schuster, 1955), por lo que la variación genética que poseen es reducida.

\section{Experimento III.}

El análisis de varianza mostró diferencias significativas entre las cepas, entre los genotipos y en la interacción cepa por genotipos $(\mathrm{P}<0,05)$ (Cuadro 3). La presencia de interacciones genotipo por cepa sugiere la existencia de resistencia de tipo vertical entre los genotipos evaluados (Van der Plank, 1984; Robinson, 1987). También se observaron genotipos con diferentes niveles de resistencia de tipo horizontal.

Las cepas de $X c p$ mostraron distinta capacidad patogénica e infectaron diferente número de genotipos mejorados del Programa de Frijol del CIAT. Se identificaron cepas muy patogénicas procedentes de la Mesa Central, como la Mex-95 y la Mex-99, que fueron compatibles con cinco de los siete genotipos inoculados (Cuadro 3). La cepa Mex-13 sólo fue compatible con la variedad XAN 266 (Cuadro 3), las demás cepas indujeron diferente reacción en los genotipos evaluados.

La mayoría de los genotipos que mostraron resistencia en este experimento, excepto A 475, A 36 y G 11867 se utilizaron en la evaluación de la variación patogénica de siete cepas de $X c p$, inoculadas cada una por separado y donde MCD 4012 fue resistente a todas las cepas evaluadas, como se observa en el Cuadro 6.

Todas las cepas de $X c p$ tuvieron una patogenicidad leve en la variedad MCD 4012, por lo que se puede considerar que ésta presenta resistencia de tipo horizontal (Robinson, 1987). En otros genotipos evaluados las cepas mostraron diferentes grados de patogenicidad y provocaron distintas reacciones de resistencia, lo que indica una probable resistencia de tipo vertical (Van der Plank, 1984).

Todas las cepas de $X c p$ evaluadas indujeron una patogenicidad severa en la variedad XAN 266, mientras que los genotipos REN 27 y SEA 14, sólo fueron compatibles con dos de las siete cepas empleadas en este experimento. Estos genotipos junto con la MCD 4012 podrían utilizarse como progenitores en programas de mejoramiento para resistencia al tizón común del frijol.

\section{DISCUSIÓN}

La variación en la patogenicidad de la bacteria interfiere con el desarrollo de variedades con resistencia vertical o específica, por lo que es importante identificar progentiores con resistencia a un amplio rango de patogenicidad de Xcp para obtener variedades con resistencia durable o con adaptación a diferentes ambientes. Las variedades con resistencia vertical o específica, podrían utilizarse en localidades específicas en combinación con otras medidas para el control de la enfermedad.

La variación patogénica de $X c p$ dificulta el desarrollo de genotipos resistentes de amplia adaptación. Por ejemplo, genotipos resistentes desarrollados en el Programa de Frijol de la Universidad de Nebraska y en el del CIAT, resultaron susceptibles cuando se cultivaron en áreas diferentes a donde fueron desarrollados (Ekpo y Saettler, 1976). De la misma forma sucedió cuando se inocularon algunos de estos genotipos con cepas de Xcp de México (Cuadros 2 y 3).

Probablemente en estos genotipos existe un fuerte componente de resistencia vertical, el cual se inactiva cuando las plantas consideradas como "resistentes" se exponen a cepas del patógeno diferentes en patogenicidad a las utilizadas durante su desarrollo. Por ello, es

Cuadro 3. Reacción de siete genotipos mejorados de frijol a siete cepas de Xanthomonas campestris pv. phaseoli en invernadero. México, 1995.

\begin{tabular}{|c|c|c|c|c|c|c|c|}
\hline \multirow[t]{3}{*}{ Genotipos } & \multicolumn{7}{|c|}{ Cepas } \\
\hline & \multicolumn{2}{|c|}{ Edo. de México } & \multirow{2}{*}{$\begin{array}{c}\text { Chihuahua } \\
44\end{array}$} & \multirow{2}{*}{$\begin{array}{c}\text { Aguascalientes } \\
0,1\end{array}$} & \multicolumn{2}{|c|}{ Durango } & \multirow{2}{*}{$\begin{array}{c}\text { Puebla } \\
95\end{array}$} \\
\hline & 13 & 99 & & & 27 & 24 & \\
\hline MCD 4011 & $3,0 *$ & 3,5 & 3,2 & 4,0 & 2,9 & 4,1 & 3,3 \\
\hline MCD 4012 & 2,7 & 2,9 & 2,9 & 2,9 & 1,7 & 2,9 & 2,7 \\
\hline REN 27 & 2,1 & 2,5 & 3,2 & 2,2 & 3,0 & 3,4 & 2,0 \\
\hline SEA 14 & 1,5 & 3,5 & 1,9 & 2,4 & 2,9 & 3,0 & 3,2 \\
\hline Harowood & 2,5 & 3,9 & 5,2 & 5,2 & 3,8 & 2,7 & 5,2 \\
\hline G 5686 & 2,4 & 3,9 & 2,9 & 3,8 & 2,3 & 3,5 & 3,7 \\
\hline XAN 266 & 5,4 & 6,0 & 5,9 & 5,9 & 3,2 & 5,0 & 3,8 \\
\hline
\end{tabular}

* media de diez repeticiones, 1-3 resistente, 4-6 intermedio y 7-9 susceptible, inoculación por corte con navajas. 
importante utilizar un amplio rango de patogenicidad de $X c p$ en los programas de mejoramiento genético del frijol, o bien utilizar la técnica del patotipo designado para la identificación de genotipos con resistencia de tipo horizontal (Robinson, 1987). Esta última técnica consiste en utilizar una cepa del patógeno que sea capaz de acoplar al mayor número de genotipos del hospedante que se vayan a evaluar.

Los resultados obtenidos en esta investigación son similares a los señalados por otros autores (Schuster et al., 1984; Schuster, 1983; Yoshii et al., 1978; Ekpo y Saettler, 1976), acerca de que los genotipos considerados como resistentes en una región o país, se muestran susceptibles a las cepas de Xcp en otro lugares. Esto puede deberse, como ya se mencionó, a la variación en la patogenicidad de la bacteria y a la probable resistencia de tipo vertical presente en estos genotipos. Además, las diferentes condiciones ambientales pueden modificar la acción de los genes de resistencia (Arnaud-Santana et al., 1994).

La coexistencia patógeno-hospedante permite la activación de mecanismos de resistencia en el hospedante y de patogenicidad en el patógeno. Para que pueda presentarse la reacción de compatibilidad, el patógeno necesita tener los genes que le permitan vencer la resistencia del hospedante e inducir el acoplamiento (Robinson, 1987). En el caso del germoplasma introducido, como no ha tenido contacto con las cepas del patógeno originarias de México, probablemente puedan mostrar una mayor resistencia o mayor susceptibilidad a ellas. En el caso de la resistencia de genotipos introducidos, ésta podría ser más duradera porque el patógeno tendría que adaptarse para contar con genes útiles para vencer esta resistencia.

En áreas de amplia variación patogénica donde se han utilizado genotipos con genes de resistencia específicos a una raza de un patógeno, la resistencia pocas veces ha sido durable (Price, 1980; Parlevliet, 1993). Price (1980) calculó la velocidad de cambio en la resistencia del hospedante, mediante la exposición de poblaciones del patógeno a hospedantes con un factor de resistencia. Después de siete a diez generaciones, la población del patógeno pudo modificar su capacidad para tolerar el factor de resistencia. Cuando utilizó hospedantes en los que se combinaron seis factores de resistencia, la población del patógeno no modificó su capacidad de patogenicidad en 30 generaciones.

Por lo tanto, si se combinaran mas factores de resistencia en el hospedante, la población del patógeno podría retardar su velocidad de cambio y el hospedante conservaría su resistencia por mayor tiempo. Además, la acumulación de genes de resistencia parcial ha servido para reducir la tasa de cambio del patógeno, esto se ha observado principalmente en aquellos patógenos que tienen reproducción asexual, como las bacterias (Price, 1980). Otra forma de retrasar la velocidad de cambio en la patogenicidad y de lograr una resistencia con mayor duración es la obtención de genotipos con resistencia horizontal, resistencia que aparentemente está presente en algunos de los genotipos evaluados.

Es probable que en los genotipos resistentes identificados, como son de diferente origen, existan diferentes mecanismos y genes de resistencia, los que se podrían piramidar o acumular para lograr una resistencia más duradera. Para facilitar el desarrollo rápido de genotipos de frijol con resistencia a $X c p$, se sugiere la identificación y uso de progenitores resistentes por área agroecológica y la utilización de técnicas de inoculación para evitar escapes. Las poblaciones segregantes deben ser evaluadas por inoculación artificial con cepas virulentas de la bacteria y familias derivadas de diferentes progrenitores. Podrían recombinarse para incrementar las posibilidades de desarrollar líneas con resistencia duradera.

\section{CONCLUSIONES}

Se identificaron genotipos de frijol resistentes a cepas de Xcp aisladas de México, inoculadas con mezclas o en forma individual, entre éstos REN 27, MCD 4012 y SEA 14, resultaron sobresalientes.

Se observaron interacciones genotipo por cepa, al inocular cada cepa de Xcp en siete genotipos de frijol.

La inoculación con mezclas de cepas de Xcp resultó eficiente en la identificación de genotipos de frijol con resistencia al tizón común.

\section{AGRADECIMIENTOS}

Los autores agradecen a la FES-Cuautitlán, UNAM, por el apoyo financiero, a través de la Cátedra "Interacciones microorganismos-plantas y asociaciones derivadas de ellas".

\section{LITERATURA CITADA}

ACOSTA, G. J. A. 1992. El cultivo del frijol y su problemática en México. In. Solórzano, V. E. (ed.). Ciclo de seminarios del curso deproducción de leguminosas de grano. Universidad Autónoma Chapingo. Depto de Fitotecnia. pp. 1-11 
ARNAUD-SANTANA, E.; COYNE,D.; ESKRIDGE,K.; VIDAVER,A. 1994. Inheritance; low correlations of leaf, pod and seed reactions to common blight disease in common beans, and implications for selection. J. Amer. Soc. Hort. Sci. 119(1):116-121

CAMPOS, A. J. 1987. Enfermedades del frijol. Ed. Trillas. México, D. F. 132 p.

CIAT (Centro Internacional de Agricultura Tropical). 1984. Informe Anual del Programa de Frijol 1983. Cali, Colombia. pp. 18-43

CIAT (Centro Internacional de Agricultura Tropical). 1988. Informe Anual del Programa de Frijol 1987. Documento de trabajo No.47. Cali, Colombia. pp. 88-123

CIAT. 1987. Sistema estándar para la identificación de germoplasma de frijol. Schoonhoven, A. V. Y A. PastorCorrales (comps.). Centro Internacional de Agricultura Tropical (CIAT). Cali, Colombia. 56 p.

COYNE, D. P.; SCHUSTER,M. 1973. Phaseolus germplasm tolerant to common blight bacterium (Xanthomonas phaseoli). Plan Dis. Reptr. 57 :111-114

CRISPÍN, M. A.; SIFUENTES,J.; CAMPOS,J. 1976. Folleto de divulgación No. 39. INIA. SAG. México, D. F. 42 p.

EKPO, E. J.; SAETTLER,A. 1976. Pathogenic variation in Xanthomonas phaseoli and Xanthomonas phaseoli var. fuscans. Plant Dis. Reptr. 60 : 80-83

FREED, R.; EISENSMITH,S., GOETZ,S.; REICOSKY,D.; SMAIL,V.; WOLBERG,P. 1991. MSTAT-C. A microcomputer program for design, management, and analysis of agronomic research experiments. Michigan State University.

GILBERTSON, R. L.; MAXWELL,D. 1992. Common bacterial blight of bean. In: Chaube, H. S., U. S. Singh, A. N. Mukhopadhyay, and J. Kumar (eds.) Plant diseases of international importance. Vol. II. Diseases of vegetables and oil seed crops. Prentice Hall. New Jersey, N. J. pp. 18-39.

KIRALY, Z.; KLEMENT,Z.; SOLYMOSY,F.; VÖROS,J. 1970. Methods in plant pathology. Ed. Akàdemiai Kiado. Budapest, Hungary. 509 p.

LÉPIZ, R. 1979. Informe 1977. Programa Nacional de Frijol. INIA. SARH. 233 p.

LÓPEZ, F. 1991. Definición de prioridades de investigación fitopatológica para la zona templada del Altiplano Central de México.Agric. Téc. Méx. 17(1y 2):17-51
PARLEVLIET, J. E . 1993. What is durable resistance, a general outline. In: Durability of disease resistance. (Jacobs, Th. and Parlevliet, J. E. eds.). Kleuver Acad. Publ. Dordrecht, The Netherlands. p

PASTOR, M. A.; BEEBE,S.; CORREA,F. 1981. Comparing 2 techniques for evaluating resistance in beans to Xanthomonas campestris pv. phaseoli. Proc. Frith Int. Conf. Plant Path. Bact. Cali, Colombia. pp. 493-503

PRICE, P. W. 1980. Evolutionary biology of the parasites. Monographs in population biology. Princeton Univ. Press. Princeton, N. Jersey. 237 p.

ROBINSON, R. A. 1987. Host management in crop pathosystems. MacMillan Pub. Co. New York. 263 p.

RODRÍGUEZ, M. A.; RAMÍREZ,H.; VICTORIA,M.; VOYSEST,O.;WHITE,J. 1995. Catalog of advanced bean lines from CIAT. Centro Internacional de Agricultura Tropical (CIAT). Cali, Colombia. 285 p.

SAETTLER, A. W. 1991. Diseases caused by bacteria. In. Hall, R. (ed.). Compendium of bean diseases. APS Press. St. Paul, Minn. pp. 29-30

SHEPPARD, J. W.; ROTH,D.; SAETTLER,A. 1989. Detection of Xanthomonas campestris pv. phaseoli in bean. In. A. W. Saettler, N. W Schaad, and D. A. Roth (eds.). Detection of bacteria in seed and other planting material. APS Press. St. Paul, Minn. pp. 17-29.

SCHUSTER, M. L. 1955. A method for testing resistance of beans to bacterial blights. Phytopathology 45(9):519-520.

SCHUSTER, M. L.; COYNE,D.; HOFF,B. 1973. Comparative virulence of Xanthomonas phaseoli strains from Uganda, Colombia and Nebraska. Plant Dis. Reptr. 57:74-75.

SINGH, S. P. 1992. Common bean improvement in the tropics. In. Plant Breeding Rev. Vol 10. 199-269.

VALLE, P.; OCHOA,R.; RAMOS,F.; VILLALOBOS,J. 1987. Evaluación cuantitativa de pérdidas por enfermedades de frijol de temporal en Aguascalientes. XIV Congreso Nacional de Fitopatología. Soc. Mex. Fitopat. Morelia, Mich. p. 92

VAN DER PLANK, J. E. 1984. Disease resistance in plants. 2nd Ed. Acad. Press, Inc. Orlando, Fla. 194 p.

YOSHII, K.; GÁLVEZ,G.; ALVAREZ,G. 1978. Screening bean germplasm for tolerance to Common Blight caused by Xanthomonas phaseoli and the importance of pathogenic variation to varietal improvement. Plant Dis. Reptr. 62 :343-347. 